\title{
FINITE GROUPS WITH TWO $p$-REGULAR CONJUGACY CLASS LENGTHS II
}

\author{
ELENA ALEMANY, ANTONIO BELTRÁN ${ }^{凶}$ and MARÍA JOSÉ FELIPE
}

\author{
(Received 16 June 2008)
}

\begin{abstract}
Let $G$ be a finite group. We prove that if the set of $p$-regular conjugacy class sizes of $G$ has exactly two elements, then $G$ has Abelian $p$-complement or $G=P Q \times A$, with $P \in S y l_{p}(G), Q \in S y l_{q}(G)$ and A Abelian.
\end{abstract}

2000 Mathematics subject classification: primary 20E45; secondary 20Dxx.

Keywords and phrases: finite-groups, $p$-regular elements, conjugacy class sizes.

\section{Introduction}

Itô proved in [5] that if $G$ is a finite group such that all its noncentral conjugacy classes have equal size, then $G=Q \times A$, where $Q$ is a Sylow $q$-subgroup of $G$, for some prime $q$, and $A$ lies in $\mathbf{Z}(G)$. In [1], Beltrán and Felipe proved a generalization of this result for $p$-regular conjugacy class sizes and some prime $p$, with the assumption that the group $G$ is $p$-solvable. In the present paper, we improve this result by showing that the $p$-solvability condition is not necessary.

THEOREM A. Let $G$ be a finite group. If the set of p-regular conjugacy class sizes of $G$ has exactly two elements, for some prime $p$, then $G$ has Abelian p-complement or $G=P Q \times A$, with $P \in S y l_{p}(G), Q \in S y l_{q}(G)$ and $A \subseteq \mathbf{Z}(G)$, with $q$ a prime distinct from $p$. As a consequence, if $\{1, m\}$ are the $p$-regular conjugacy class sizes of $G$, then $m=p^{a} q^{b}$. In particular, if $b=0$ then $G$ has Abelian $p$-complements and if $a=0$ then $G=P \times Q \times A$ with $A \subseteq \mathbf{Z}(G)$.

The proof given in [1] for $p$-solvable groups is divided into two cases, when the centralizers of noncentral $p$-regular elements are all $G$-conjugated and when they are not. In the second case, it is easy to check that the hypothesis of $p$-solvability is not needed, so our study reduces then to the case in which all the centralizers of noncentral $p$-regular elements are conjugated. In order to solve this case, we are going to base

This work was partially supported by grant MTM2007-68010-C03-03 and the second author is also supported by grant Fundació Caixa-Castelló P1-1A2006-06.

(C) 2009 Australian Mathematical Society 0004-9727/2009 \$16.00 
our arguments on the proof of a theorem of Camina [2, Theorem 1]. We stress that while Camina used the classification obtained by Gorenstein and Walter [3] of those groups whose Sylow 2-subgroups are dihedral (this having been used to complete the classification of the simple finite groups), we present a more simple proof by making use of a well-known theorem of Kazarin which asserts that in any finite group the subgroup generated by an element of prime power class size is always solvable [4, Theorem 15.7].

Furthermore, we remark that it is not feasible that all the centralizers of noncentral elements of a group $G$ are conjugate, but it is easy to find examples where all the centralizers of noncentral $p$-regular elements are conjugate (consequently $G$ has exactly two $p$-regular conjugacy class sizes) for some prime $p$. For instance, the centralizers of all noncentral 2-elements of $\operatorname{SL}(2,3)$ are conjugate and the 3-regular class sizes are $\{1,6\}$. Another example is Alt(4), whose 2-regular class sizes are $\{1,4\}$.

We shall assume that every group is finite and we shall denote by $G_{p^{\prime}}$ the set of $p$-regular elements of $G$.

\section{Preliminary results}

We shall need some results on conjugacy classes of $p$-regular elements.

LEMMA 1. Let $G$ be a finite group. Then all the conjugacy class sizes in $G_{p^{\prime}}$ are p-numbers if and only if $G$ has Abelian p-complements.

Proof. See [1, Lemma 2].

The following is exactly [2, Lemma 1], but we present an easier proof. It generalizes [1, Lemma 3 ] by eliminating the hypothesis of $p$-solvability.

LEMMA 2. Suppose that $G$ is a finite group and that $p$ is not a divisor of the sizes of p-regular conjugacy classes. Then $G=P \times H$ where $P$ is a Sylow p-subgroup and $H$ is a p-complement of $G$.

PROOF. Let $g \in G$ and consider its $\left\{p, p^{\prime}\right\}$-decomposition as $g=g_{p} g_{p^{\prime}}$. Suppose that $g_{p^{\prime}}$ is noncentral. As the class size of $g_{p^{\prime}}$ is a $p^{\prime}$-number, if we fix a Sylow $p$-subgroup $P$ of $G$, then there exists some $t \in G$ such that $g_{p} \in P^{t} \subseteq C_{G}\left(g_{p^{\prime}}\right)$. Therefore,

$$
G=\bigcup_{t \in G} P^{t} C_{G}\left(P^{t}\right)
$$

Then $G=P C_{G}(P)$ and so, $G=P \times H$ where $H$ is a $p$-complement of $G$.

LeMma 3. Let $P$ be an Abelian $p$-group, with $p$ a prime and let $K$ be a group of automorphisms of $P$ such that $|K|$ is divisible by $p$. Suppose that $C_{P}(x)=C_{P}(y)$ for all $x, y \in K-\{1\}$. Then $\mathbf{O}_{p^{\prime}}(K)=1$.

Proof. Assume that $H=\mathbf{O}_{p^{\prime}}(K)>1$ and we shall get a contradiction. Suppose first that $C_{P}(H)=1$ and take some nontrivial $x \in H$. If there exists some element 
$w \in C_{P}(x)-\{1\}$, then clearly $w \in C_{P}(H)$ and so, necessarily, $C_{P}(x)=1$ and hence, $C_{P}(y)=1$ for all $y \in K-\{1\}$. But if we count the orbit sizes this cannot happen because $p$ divides $|K|$.

As a result, $C_{P}(H) \neq 1$. Now, as $P$ is Abelian, by coprime action we can write $P=C_{P}(H) \times[P, H]$, and since $C_{P}(K)=C_{P}(H)$ and $K$ is a group of automorphisms of $P$, it follows that $[P, H] \neq 1$. Thus, if $x \in K-\{1\}$, then $C_{P}(x)=$ $C_{P}(K) \times C_{[P, H]}(x)$. Now, if $w \in C_{[P, H]}(x)-\{1\}$, then $C_{P}(w)=C_{P}(K)$, so $w \in$ $C_{P}(K) \cap[P, H]=1$. This is not possible, so $C_{[P, H]}(x)=1$ for all $x \in K-\{1\}$. But this contradicts again the fact that $p$ is a divisor of $|K|$.

LEMMA 4. Let $G$ be a finite group such that all its Sylow subgroups are cyclic. If $r$ and $s$ are two distinct primes dividing $|G|$, then there exists a subgroup $U$ of $G$ such that $|U|=r s$.

PROOF. We work by induction on the order of $G$. First, it is known that any finite group whose Sylow subgroups are all cyclic is solvable (see for instance [6, 10.1.10]). Let $M$ be a maximal normal subgroup of $G$, so $|G: M|=p$ for some prime $p$. We can assume that $M$ is a $p^{\prime}$-subgroup, otherwise we can apply the inductive hypothesis to $M$ and the result is obtained. Also, we only have to show that there exists a subgroup of order $p q$ for any prime $q \neq p$ dividing $|M|$, since the other cases are obtained by the inductive hypothesis as well. If $P$ is a Sylow $p$-subgroup of $G$, then $P$ acts coprimely on $M$, so if we fix a prime $q$ dividing $|M|$, we know (see for example $[4,14.3]$ ) that there exists some $P$-invariant Sylow $q$-subgroup $Q$ of $G$, which is cyclic. Hence, if $x \in Q$ has order $q$, then $U=\langle x\rangle P$ has order $p q$, as required.

\section{Proof of Theorem A}

We shall prove by induction on the order of $G$ that either $G$ has Abelian $p$ complements or $G$ is a $\{p, q\}$-group for some prime $q \neq p$ without considering central factors. Likewise, we notice that when $G$ is solvable then the theorem is already proved by [1, Theorem A]. We shall assume then that the $p$-complements of $G$ are not Abelian and that there exist at least two prime divisors of the order of $G / \mathbf{Z}(G)$ different from $p$, in order to get a contradiction.

As we have already pointed out in the introduction, we are also going to assume that all the centralizers of noncentral elements in $G_{p^{\prime}}$ are conjugated in $G$. In the other case the theorem can be proved exactly the same as case 2 of [1, Theorem A], where the condition of $p$-solvability is not necessary. More precisely, the conjugation of the centralizers of all noncentral elements in $G_{p^{\prime}}$ will be used from Step 4.

The first two steps are exactly Steps 1 and 4 of [1, Theorem A], so we shall omit their proofs.

STEP 1. We can assume that $C_{G}(x)=P_{x} \times L_{x}$, with $P_{x}$ a Sylow $p$-subgroup of $C_{G}(x)$ and $L_{x} \leq Z\left(C_{G}(x)\right)$, for any noncentral $x \in G_{p^{\prime}}$.

STEP 2. $C_{G}(x)<N_{G}\left(C_{G}(x)\right)$ for every noncentral $x \in G_{p^{\prime}}$. 
STEP 3. If $x \in G_{p^{\prime}}$ is noncentral, then every Sylow subgroup of $N_{G}\left(C_{G}(x)\right) / C_{G}(x)$ is cyclic or generalized quaternion. Furthermore, if $q \neq p$ is a prime divisor of the order of this group, then the Sylow $q$-subgroup has order $q$.

We fix some $x \in G_{p^{\prime}}$ and write $W=N_{G}\left(C_{G}(x)\right) / C_{G}(x)$. Let $Q$ be a Sylow $q$ subgroup of $W$ for some prime $q$ dividing $|W|$ (possibly $q=p$ ). By the assumptions we have made at the beginning of the proof there exists some prime $r$, divisor of $|G / \mathbf{Z}(G)|$, distinct from $q$ and $p$. Clearly $r$ divides $\left|C_{G}(x)\right|$ since all these centralizers have the same size. Let $R_{x}$ be a Sylow $r$-subgroup of $C_{G}(x)$ and notice that $Q$ acts as a permutation group on $R_{x}$ since if $g \in Q$, then $C_{R_{x}}(g)=R_{x} \cap \mathbf{Z}(G)$. Moreover, since this is a coprime action and $R_{x}$ is Abelian, we can write $R_{x}=\left[R_{x}, Q\right] \times C_{R_{x}}(Q)$. Also, observe that $Q$ acts fixed-point-freely on $\left[R_{x}, Q\right]$, for if $t \in\left[R_{x}, Q\right]-\{1\}$, then $C_{G}(t)=C_{G}(x)$ by Step 1 , so no element of $Q-\{1\}$ may fix $t$. Consequently, we can apply a well known result ([4, Theorem 16.12] for instance) to obtain that $Q$ is cyclic or generalized quaternion.

Assume now that $q \neq p$ and take $Q_{x}$ a Sylow $q$-subgroup of $C_{G}(x)$, which is normal by Step 1. Accordingly, $Q$ acts on $\bar{Q}_{x}=Q_{x} / \mathbf{Z}(G)_{q}$. If $M$ is the semidirect product defined by this action, we can take some element in $\mathbf{Z}(M) \cap \bar{Q}_{x}$ which has exactly order $q$. If $\bar{t} \in \bar{Q}_{x}$, with $t \in Q_{x}$ is such an element, we can construct the subgroup $T=\langle t\rangle \mathbf{Z}(G)_{q} \leq C_{G}(x)$. Observe that $Q$ acts faithfully on $T$, that is, $C_{Q}(T)=1$, since $C_{G}(t)=C_{G}(x)$ by Step 1 . Furthermore, notice that $[T, Q] \subseteq$ $Z(G)_{q}$. We claim now that $Q$ is a $q$-elementary subgroup. Let $v \in Q$. As $t^{q} \in \mathbf{Z}(G)$, then $1=\left[t^{q}, v\right]=[t, v]^{q}$, where the last equality follows because $T$ is Abelian. Also, since $[t, v] \in \mathbf{Z}(G)$ we have $[t, v]^{q}=\left[t, v^{q}\right]$, so we conclude that $v^{q} \in C_{Q}(T)=1$ and thus $Q$ is elementary, as claimed. But this implies that $Q$ is cyclic of order $q$ by the above paragraph, and hence the step is proved.

STEP 4. For any $x \in G_{p^{\prime}}$, we have $\left|N_{G}\left(C_{G}(x)\right) / C_{G}(x)\right|=q$ for some fixed prime $q \neq p$.

First we are going to prove that $W=N_{G}\left(C_{G}(x)\right) / C_{G}(x)$ is $q$-group for some prime $q$ (including the possibility $q=p$ ). Suppose that $|W|$ is divisible by at least two distinct primes and we shall prove that there exists a subgroup $U$ of $W$ such that $|U|$ is the product of two prime numbers. By Step 3, if every Sylow subgroup of $W$ is cyclic then there exists such subgroup $U$ by Lemma 4 . We can assume then that 2 divides $|W|$ and that the Sylow 2-subgroups of $W$ are generalized quaternion, so we can apply a classic theorem of Brauer and Suzuki (see [4, 45.1]) to obtain that $\mathbf{O}_{2^{\prime}}(W)\langle\tau\rangle \unlhd W$, where $\tau$ is an involution of $W$. Again by Step 3, the Sylow subgroups of $\mathbf{O}_{2^{\prime}}(W)$ are cyclic, so if $\left|\mathbf{O}_{2^{\prime}}(W)\right|$ is divisible by at least two distinct primes then the subgroup $U$ exists by Lemma 4 as well. So we can suppose that $\mathbf{O}_{2^{\prime}}(W)$ is a cyclic $r$-group for some prime $r \neq 2$. Hence we can take $\alpha \in \mathbf{O}_{2^{\prime}}(W)$ of order $r$ and we may construct the subgroup $U=\langle\alpha\rangle\langle\tau\rangle$ of order $2 r$. As a result, in all the cases we have a subgroup $U \leq W$ such that $|U|=r s$, for some primes $r$ and $s$, as we wanted to prove. We shall see now that this leads to a contradiction. If both primes are distinct from $p$, then either $U$ has a normal $r$-complement or has 
a normal $s$-complement, and we shall assume without loss that the $r$-complement is normal. In the other case, that is, if $|U|=p r$, with $r \neq p$ then, arguing as in the first paragraph of Step 3, we get that $U$ operates as a permutation group and fixed-pointfreely on $\left[S_{x}, U\right]-1$, where $S_{x}$ is the Sylow $s$-subgroup of $C_{G}(x)$ for some prime $s \notin\{p, r\}$. Notice that such $s$ exists by the assumption we have made at the beginning. Furthermore, in this second case (by applying for instance [4, Lemma 16.12]) we get that $U$ is cyclic, so in particular, $U$ has nontrivial normal $r$-complement. Thus, in both cases, $U$ has a normal $r$-complement for some prime $r \neq p$. However, $U$ is an automorphism group of $R_{x}$, where $R_{x}$ is the Abelian Sylow $r$-subgroup of $C_{G}(x)$. Moreover, if $u, v \in U-\{1\}$, then $C_{R_{x}}(u)=C_{R_{x}}(v)=\mathbf{Z}(G)_{r}$, so by Lemma 3, we get $\mathbf{O}_{r^{\prime}}(U)=1$, which is a contradiction.

Take now a noncentral Sylow $r$-subgroup $R_{x}$ of $C_{G}(x)$, for some prime $r \neq p$. If $t \in R_{x}$ is noncentral, then by applying Step 1 , we have $C_{G}(x)=C_{G}(t)$. If $w \in$ $N_{G}\left(R_{x}\right)$, then by the same reason, $C_{G}\left(t^{w}\right)=C_{G}(t)$. Therefore, $C_{G}(x)=C_{G}(t)^{w}=$ $C_{G}(x)^{w}$ and $w \in N_{G}\left(C_{G}(x)\right)$. Thus $N_{G}\left(R_{x}\right) \leq N_{G}\left(C_{G}(x)\right)$. Nevertheless, notice that if $R_{x}$ is not a Sylow $r$-subgroup of $G$, then $R_{x}<N_{G}\left(R_{x}\right)$, so $r$ divides $\left|N_{G}\left(R_{x}\right) / R_{x}\right|$, and this implies that $|W|$ is divisible by $r$, so $W$ cannot be a $p$-group. By taking into account Step 3, the step is proved.

The fact that all the centralizers are conjugated implies that we can assume for the rest of the proof that $\left|N_{G}\left(C_{G}(x)\right) / C_{G}(x)\right|=q$, for a fixed prime $q \neq p$ and for any noncentral $x \in G_{p^{\prime}}$.

STEP 5. We can assume that $\mathbf{O}_{p}(G)=1$ and that $\left|G: N_{G}\left(C_{G}(x)\right)\right|$ is a $p$-number for any noncentral $x \in G_{p^{\prime}}$.

We fix a noncentral $x \in G_{p^{\prime}}$ and for any prime $r \neq p$ we take $R$ a Sylow $r$-subgroup of $G$. If $R$ is Abelian, as all the centralizers of noncentral elements in $G_{p^{\prime}}$ have the same order, then the Sylow $r$-subgroup of $C_{G}(x), R_{x}$, is a Sylow $r$-subgroup of $G$ and $R$ is conjugated to $R_{x}$. Thus, $r$ does not divide $\left|G: N_{G}\left(C_{G}(x)\right)\right|$. If $R$ is not Abelian, then it is an elementary fact that there exists some $t \in R-\mathbf{Z}(R)$ such that $C_{R}(t) \unlhd R$. As the centralizers of all noncentral $p$-regular elements are conjugate, we can assume without loss that $C_{G}(t)=C_{G}(x)$. In particular, $C_{R}(t) \subseteq$ $C_{G}(x)$. On the other hand, is $g \in N_{G}\left(C_{R}(t)\right)$, then $t^{g} \in C_{R}(t)$ and $C_{G}(t)=$ $C_{G}\left(t^{g}\right)$ by Step 1. Consequently, $C_{G}(x)=C_{G}(t)=C_{G}\left(t^{g}\right)=C_{G}(x)^{g}$ and so $g \in$ $N_{G}\left(C_{G}(x)\right)$. Thus $R \leq N_{G}\left(C_{R}(t)\right) \leq N_{G}\left(C_{G}(x)\right)$, and so $\left|G: N_{G}\left(C_{G}(x)\right)\right|$ is an $r^{\prime}-$ number too. Accordingly, in both cases we have proved that $\left|G: N_{G}\left(C_{G}(x)\right)\right|$ is a p-number.

Now we assume that $\mathbf{O}_{p}(G) \neq 1$ and we are going to see that $\bar{G}=G / \mathbf{O}_{p}(G)$ satisfies the hypotheses of the theorem. We fix some noncentral element $x \in G_{p^{\prime}}$. Let $\bar{y} \in C_{\bar{G}}(\bar{x})$ and notice that $[x, y] \in \mathbf{O}_{p}(G)$. Hence, we can write $x^{y}=x a$, with $a \in \mathbf{O}_{p}(G)$, so $x^{y}$ is a $p^{\prime}$-element of $C_{G}(x) \mathbf{O}_{p}(G)$, and then $x^{y} \in L_{x}^{t}$, for some $t \in \mathbf{O}_{p}(G)$, where $L_{x}$ is the $p^{\prime}$-subgroup appearing in Step 1. Therefore $x^{y t^{-1}} \in L_{x}$ and $C_{G}(x)=C_{G}\left(x^{y t^{-1}}\right)$. As a consequence, $y t^{-1} \in N_{G}\left(C_{G}(x)\right)$, so $y=w t$ with $w \in N_{G}\left(C_{G}(x)\right)$. Thus, $\bar{y}=\bar{w}$ and $\overline{w x}=\bar{x} \bar{w}$, that is, $[w, x] \in \mathbf{O}_{p}(G)$. On the other 
hand, as $w \in N_{G}\left(C_{G}(x)\right)$ and $x$ is a $p$-regular element, this forces $[w, x]$ to be a $p$ regular element, so $[x, w]=1$. Therefore, $C_{\bar{G}}(\bar{x})=\overline{C_{G}(x)}$ and we conclude that $\bar{G}$ has two class sizes of $p$-regular elements. By the inductive hypothesis, either $\bar{G}$ has an Abelian $p$-complement or $\bar{G}=\overline{P Q} \times \bar{A}$, with $\bar{P} \in S y l_{p}(\bar{G}), \bar{Q} \in S y l_{q}(\bar{G})$ and $\bar{A} \leq \mathbf{Z}(\bar{G})$. In the first case, $G$ has an Abelian $p$-complement, contradicting our first assumptions and in the second one, $G$ is a solvable group, so the proof would be finished.

STEP 6. $\mathbf{O}_{r}(G) \subseteq \mathbf{Z}(G)$, for every prime $r \neq p$.

Let $r$ be any prime distinct from $p$ and suppose that $K=\mathbf{O}_{r}(G)$ is noncentral. By Step 5, we have $K \subseteq N_{G}\left(C_{G}(x)\right)$, for all $x \in G_{p^{\prime}}$. The hypothesis and Step 1 imply that there exists an Abelian noncentral normal Sylow $s$-subgroup of $C_{G}(x)$, say $S_{x}$, for some prime $s \neq p, r$. Notice that $S_{x}$ is normalized by $K$ and thus $\left[S_{x}, K\right] \subseteq S_{x} \cap K=1$, so $K \subseteq C_{G}\left(S_{x}\right)=C_{G}(x)$, where the last equality follows as a consequence of Step 1. On the other hand, if $t \in K-\mathbf{Z}(G)$, then $C_{G}(t)=C_{G}(x)$ again by Step 1. Moreover, if $w \in N_{G}(K)$, then $C_{G}\left(t^{w}\right)=C_{G}(x)$, hence $C_{G}(x)^{w}=$ $C_{G}(t)^{w}=C_{G}\left(t^{w}\right)=C_{G}(x)$. Thus, $G=N_{G}(K) \subseteq N_{G}\left(C_{G}(x)\right)$ and $C_{G}(x) \unlhd G$. By Step 4, we have $\left|G: C_{G}(x)\right|=q$. This means that $m=q$, so by applying Lemma 2 and Itô's theorem on groups with two conjugacy class sizes (see for instance [4, Theorem 33.6]), we obtain $G=P \times Q \times A$, with $P \in \operatorname{Syl}_{p}(G), Q \in \operatorname{Syl}_{q}(G)$ and $A$ Abelian, against our initial assumption.

STEP 7. We can now derive the conclusion.

First, we notice that $\mathbf{Z}(G)_{q} \neq 1$, since any element lying in the centre of a Sylow $q$-subgroup of $G$ must be central in $G$ too because $q$ divides $m$ by Step 4. We write $\bar{G}=G / \mathbf{Z}(G)_{q}$ and we shall prove that $\bar{G}$ has two $p$-regular conjugacy class sizes.

We can trivially assume that $\bar{G}$ is not Abelian, otherwise $G$ would be solvable and the proof is finished. If $\bar{a} \in \bar{G}-\mathbf{Z}(\bar{G})$, we observe that $\overline{C_{G}(a)} \subseteq C_{\bar{G}}(\bar{a})$. If $\overline{C_{G}(a)}=$ $C_{\bar{G}}(\bar{a})$ for all $\bar{a} \in \bar{G}-\mathbf{Z}(\bar{G})$, it certainly follows that $\bar{G}$ has two $p$-regular conjugacy class sizes, as we wanted. Suppose then that there is a $p$-regular element $\bar{a} \in \bar{G}$ such that $\overline{C_{G}(a)} \neq C_{\bar{G}}(\bar{a})$. It is easy to see that if $\bar{w} \in C_{\bar{G}}(\bar{a})$ then $w \in N_{G}\left(C_{G}(a)\right)$, that is, $C_{\bar{G}}(\bar{a}) \subseteq \overline{N_{G}\left(C_{G}(a)\right)}$. As $\left|\overline{N_{G}\left(C_{G}(a)\right)}: \overline{C_{G}(a)}\right|=q$ by Step 4 , this implies that $\overline{N_{G}\left(C_{G}(a)\right)}=C_{\bar{G}}(\bar{a})$ and so, by Step 5 we conclude that $\left|\bar{G}: C_{\bar{G}}(\bar{a})\right|$ is a $p$-number. Now, by a renowned theorem due to Kazarin (see for example $[4,15.7]$ ), the subgroup $\langle\bar{a} \bar{G}\rangle$ is a solvable normal subgroup of $\bar{G}$. It is easy to see then that this implies that $\left\langle a^{G}\right\rangle$ is a noncentral solvable normal subgroup of $G$ too, but this is not possible in view of Steps 5 and 6.

Therefore, we have proved that $\bar{G}$ has two $p$-regular conjugacy class sizes, and by induction we obtain that $\bar{G}$ has an Abelian $p$-complement or $\bar{G}=\overline{P Q} \times \bar{A}$, with $\bar{P} \in \operatorname{Syl}_{p}(\bar{G}), \bar{Q} \in S y l_{q}(\bar{G})$ and $\bar{A} \subseteq \mathbf{Z}(\bar{G})$. Both cases lead to the solvability of $G$, so the proof is finished.

The last assertions in the statement of the theorem will follow then by immediate application of Lemmas 1 and 2. 


\section{References}

[1] A. Beltrán and M. J. Felipe, 'Finite groups with two $p$-regular conjugacy class lengths', Bull. Aust. Math. Soc. 67 (2003), 163-169.

[2] A. R. Camina, 'Finite groups of conjugate rank 2', Nagoya Math. J. 53 (1974), 47-57.

[3] D. Gorenstein and J. H. Walter, 'On finite groups with dihedral Sylow 2-subgroups', Illinois J. Math. 6 (1962), 553-593.

[4] B. Huppert, Character Theory of Finite Groups, De Gruyter Expositions in Mathematics, 25 (Berlin, New York, 1998).

[5] N. Itô, 'On finite groups with given conjugate type I', Nagoya Math. J. 6 (1953), 17-28.

[6] D. J. S. Robinson, A Course in the Theory of Groups, Graduate Texts in Mathematics, 80, 2nd edn (Springer, New York, 1996).

ELENA ALEMANY, Departamento de Matemática Aplicada, Universidad Politécnica de Valencia, 46022 Valencia, Spain

e-mail: ealemany@mat.upv.es

ANTONIO BELTRÁN, Departamento de Matemáticas, Universidad Jaume I, 12071 Castellón, Spain

e-mail: abeltran@mat.uji.es

MARÍA JOSÉ FELIPE, Instituto Universitario de Matemática Pura y Aplicada, Universidad Politécnica de Valencia, 46022 Valencia, Spain

e-mail: mfelipe@mat.upv.es 